\title{
Leptin administered in physiological or pharmacological doses does not regulate circulating angiogenesis factors in humans
}

\author{
K. N. Aronis • K. N. Diakopoulos • C. G. Fiorenza • \\ J. P. Chamberland • C. S. Mantzoros
}

Received: 20 April 2011 /Accepted: 26 April 2011 / Published online: 10 June 2011

(C) Springer-Verlag 2011

\begin{abstract}
Aim/hypothesis Leptin has been shown to regulate angiogenesis in animal and in vitro studies by upregulating the production of several pro-angiogenic factors, but its role in regulating angiogenesis has never been studied in humans.

Methods The potential angiogenic effect of two doses of metreleptin (50 and $100 \mathrm{ng} / \mathrm{ml}$ ) was evaluated in vitro, using a novel three-dimensional angiogenesis assay. Fifteen healthy, normoleptinaemic volunteers were administered both a physiological $(0.1 \mathrm{mg} / \mathrm{kg})$ and a pharmacological $(0.3 \mathrm{mg} / \mathrm{kg})$ single dose of metreleptin, in vivo, on two different inpatient admissions separated by 1-12 weeks. Serum was collected at $0,6,12$ and $24 \mathrm{~h}$ after metreleptin administration. Twenty lean women, with leptin levels $<5 \mathrm{ng} / \mathrm{ml}$, were randomised in a 1:1 fashion to receive either physiological replacement doses of metreleptin $(0.04-0.12 \mathrm{mg} / \mathrm{kg}$ q.d.) or placebo for 32 weeks. Serum was collected at $0,8,20$ and 32 weeks after randomisation. Proteomic angiogenesis array analysis was performed to
\end{abstract}

K. N. Aronis $\cdot$ K. N. Diakopoulos $\cdot$ C. G. Fiorenza $\cdot$

J. P. Chamberland · C. S. Mantzoros

Division of Endocrinology, Diabetes and Metabolism,

Beth Israel Deaconess Medical Center, Harvard Medical School,

330 Brookline Avenue,

Boston, MA 02215, USA

C. S. Mantzoros

Section of Endocrinology, Boston VA Healthcare System,

Harvard Medical School,

Boston, MA, USA

C. S. Mantzoros $(\bowtie)$

Department of Environmental Health,

Harvard School of Public Health,

Boston, MA, USA

e-mail: cmantzor@bidmc.harvard.edu screen for angiogenic factors. Circulating concentrations of angiogenin, angiopoietin-1, platelet derived endothelial factor (PDGF)-AA, matrix metalloproteinase (MMP) 8 and 9, endothelial growth factor (EGF) and vascular EGF (VEGF) were also measured.

Results Both metreleptin doses failed to induce angiogenesis in the in vitro model. Although leptin levels increased significantly in response to both short-term and long-term metreleptin administration, circulating concentrations of angiogenesis markers did not change significantly in vivo. Conclusions/interpretations This is the first study that examines the effect of metreleptin administration in angiogenesis in humans. Metreleptin administration does not regulate circulating angiogenesis related factors in humans.

Clinical trial registration: ClinicalTrials.gov NCT00140205 and NCT00130117.

Funding: This study was supported by National Institutes of Health-National Center for Research Resources grant M01-RR-01032 (Harvard Clinical and Translational Science Center) and grant number UL1 RR025758. Funding was also received from the National Institute of Diabetes and Digestive and Kidney Diseases grants 58785, 79929 and 81913, and AG032030.

Keywords Angiogenesis · Angiogenin · Angiopoietin-1 · Humans $\cdot$ Leptin $\cdot$ MMP9
Abbreviations
ANG-1 Angiopoietin-1
CAC Circulating angiogenic cell
EGF Endothelial growth factor
MMP Matrix metalloproteinase
PDGF Platelet derived growth factor
VEGF Vascular endothelial growth factor 


\section{Introduction}

Leptin is a $16 \mathrm{kDa}$ hormone secreted primarily by the white adipose tissue. Circulating leptin concentrations are directly proportional to the amount of body fat [1] and signal information to the brain primarily on the amount of energy stored in adipose tissue and secondarily on acute alterations in energy intake [2-5]. Leptin receptors (ObRs) are widely produced in the brain and particularly in the hypothalamus and brainstem [6]. In their seminal publication, SierraHonigmann et al. demonstrate that the $\mathrm{Ob}-\mathrm{Rb}$ leptin receptor is also produced in human vasculature endothelial cells, suggesting that leptin could induce angiogenesis [7]. Although angiogenesis is required for normal growth, development and tissue repair [8], it has also been linked to a number of pathological processes, including tumour formation [9], ocular neovascularisation [10,11], rheumatoid arthritis [12], atherosclerosis [13, 14], non-alcoholic steatohepatitis (NASH) [15] and obesity [16].

A significant number of in vitro and animal studies have supported the notion that leptin could regulate angiogenesis: in vitro experiments have shown that leptin promotes endothelial tube formation $[7,17]$ and adhesion of circulating angiogenic cells (CACs) to endothelial tube structures [18]. In rodent models, leptin has been found to upregulate production of several pro-angiogenic factors, including vascular endothelial growth factor (VEGF) [19] and matrix metalloproteinases (MMPs) [20]. Although in vitro and animal studies provide highly suggestive evidence for a role of leptin in regulating angiogenesis, human studies investigating the potential regulatory role of leptin on angiogenesis and/or on levels of circulating angiogenic factors are lacking. Investigating whether leptin promotes angiogenesis in humans is essential in the era that leptin is considered for treatment of various disease states.

The purpose of this study was to investigate, for the first time in humans, whether leptin has any causal role in angiogenesis regulation. For this purpose, we administered to human participants the synthetic form of leptin, metreleptin (formerly known as met-r-Hu-Leptin), acutely (over $24 \mathrm{~h}$ ) and chronically (over 32 weeks) and evaluated the changes of the total angiogenic potential of the participants, by measuring circulating angiogenic factors, representative of all different stages of angiogenesis. To further confirm our results, we also evaluated the angiogenic potential of metreleptin with a novel HUVEC three-dimensional angiogenesis assay.

\section{Methods}

In vitro experiments

Three-dimensional HUVEC angiogenesis model We used a novel three-dimensional angiogenesis assay (promo cell, cat no. C13010) in order to screen for the effect of leptin on angiogenesis in HUVEC in vitro. In this assay, endothelial beads, consisting of 400-500 cells each, were embedded in a collagen matrix that closely resembles the base membrane. We treated the cells with two concentrations of metreleptin (50 and $100 \mathrm{ng} / \mathrm{ml}$, in culture media) and VEGF (20 ng/ml, in culture media) as a positive control, for $48 \mathrm{~h}$ at $37^{\circ} \mathrm{C}$ and $5 \% \mathrm{CO}_{2}$. As a negative control, we treated the samples with the same culture media that we used to dissolve metreleptin and VEGF (provided by the manufacturer). After treating the cells, we analysed the assay under a phase contrast microscope at $\times 10$ magnification, and expressed the angiogenic effect of the various treatments, as sprout count and cumulative sprout length in pixels. To analyse the pictures taken from the microscope, we used Image J software (NIH, NIMH, Bethesda, MD, USA).

Clinical studies and human participants

Short-term metreleptin administration study In order to evaluate the short-term effect of metreleptin administration on angiogenesis, we enrolled 15 healthy participants (five lean men, five lean women, with BMI $<25 \mathrm{~kg} / \mathrm{m}^{2}$ and five overweight men, with BMI $>27 \mathrm{~kg} / \mathrm{m}^{2}$ ), aged $18-30$ years, in an open-label study of acute metreleptin administration. A physiological dose $(0.1 \mathrm{mg} / \mathrm{kg})$ and a pharmacological dose $(0.3 \mathrm{mg} / \mathrm{kg})$ were administered to all participants on two separate overnight visits spaced by $1-12$ weeks to allow for sufficient washout [21]. The doses were selected on the basis of prior pharmacokinetics studies [22, 23]. All participants were standardised in the isocaloric fed state. Metreleptin was injected subcutaneously in the abdomen at 08:00 hours. Blood samples were taken at both visits at time 0 (immediately before the dose of metreleptin) and at times $+6 \mathrm{~h},+12 \mathrm{~h}$, and $+24 \mathrm{~h}$ after the injection to measure serum concentrations of leptin and traditional angiogenesisrelated molecules, as outlined below. In addition, serum from the baseline and $+24 \mathrm{~h}$ time points was pooled from two lean and two obese individuals who had received the $0.3 \mathrm{mg} / \mathrm{kg}$ dose of metreleptin. This serum was used to perform a proteomic array analysis to screen for a large number of angiogenesis-related factors potentially regulated by leptin, many of which are not usually evaluated in human angiogenesis studies.

Long-term metreleptin administration study In order to evaluate the long-term effect of metreleptin administration on circulating levels of angiogenic factors, we randomised 20 female participants, with leptin levels $<5 \mathrm{ng} / \mathrm{ml}$ and thus hypothalamic amenorrhoea [24, 25] (leptin sensitivity model), to receive either metreleptin $(n=11)$ or placebo $(n=9)$ for 32 weeks. The details of this study have been recently 
described [25]. Briefly, the inclusion criteria were absence of menstruation for at least 6 months, concurrent with low body weight or elevated levels of physical activity and no other cause of secondary amenorrhoea. Body weight should have been stable (within $15 \%$ of ideal body weight) for at least 6 months prior to enrolment. All participants were prescribed calcium (600 mg twice per day) and vitamin D supplements (400 IU four times per day) for the duration of the study, and were compliant with the regimen. Two participants dropped out, one on each arm; thus 10 participants receiving metreleptin and eight receiving placebo completed the study. We analysed serum samples collected at baseline and at 8, 20 and 32 weeks after randomisation. In addition to this, we performed proteomic profile array analysis on 10 participants (five on the metreleptin and five on the placebo arm) from samples that had been collected at baseline and after 32 weeks of randomisation.

All protocols were approved by the Institutional Review Board at Beth Israel Deaconess Medical Center (BIDMC). Metreleptin (Amgen, Thousand Oaks, CA, USA and subsequently Amylin, San Diego, CA, USA) was administered under an Investigational New Drug Application from the Food and Drug Administration (FDA) to C.S. Mantzoros.

\section{Experimental procedures}

Proteomic array analysis A human proteomic array kit (R\&D Systems, Minneapolis, MN, USA) was used to identify protein levels potentially regulated by leptin. Each angiogenesis array consisted of a nitrocellulose membrane containing 55 different antibodies printed in duplicate, used to detect the relative abundance of 55 different angiogenesis-related proteins. Membranes were incubated with $2 \mathrm{ml}$ blocking buffer (array buffer 7) in each well of the four-well multi-dish plate for $1 \mathrm{~h}$ on a shaker. The blocking buffer was replaced by $1 \mathrm{ml}$ of sample plus $0.5 \mathrm{ml}$ fresh array buffer 5 and incubated on the shaker overnight at $4^{\circ} \mathrm{C}$. The detection antibody cocktail and washing steps were performed as described in the manufacturer's protocol. The membranes were incubated with chemiluminescent ECL (Thermo Scientific Pierce, Waltham, MA, USA) detection reagent and exposed to $\mathrm{x}$-ray film for 1-10 min. The positive signals were quantified by Image $\mathbf{J}$ software as pixel density.

Biochemical analysis Leptin levels were measured via radioimmunoassay (LINCO Research, St Charles, MO, USA). Angiopoietin-1 (ANG-1), angiogenin, plateletderived growth factor (PDGF)-AA, MMP8, MMP9, VEGF and endothelial growth factor (EGF) concentrations were measured using ELISA (R\&D Systems). Samples were run in duplicate following the manufacturer's protocol. Oestra- diol and 25-hydroxy-vitamin D levels were analysed using radioimmunoassays (ALPCO Diagnostics, Salem, NH, USA; DiaSorin, Stillwater, MN, USA) with $\mathrm{CVs}$ and sensitivity as described by the manufacturer.

\section{Statistical analysis}

Statistical analysis was performed using Stata version 11.1 (Stata Corp., College Station, TX). Normality of the distribution for all variables was evaluated using the Shapiro-Wilk tests of normality. Variables that were not normally distributed were transformed with logarithmic or square root transformation where appropriate. Extreme outliers (deviating more than \pm 3 SDs from the mean) were excluded from the analysis. Repeated measurement ANOVA at the four time points $(0,6,12$ and $24 \mathrm{~h})$ was used for the short-term study using data from all participants. Each group (lean men $n=5$, lean women $n=5$, obese men $n=5$ ) receiving the same dose, was compared for the various analytes across the time. Post hoc comparisons between time points were performed with Tukey's HSD correction for multiple comparisons. On a second, factorial model, we examined the entire dataset with time and dose introduced in the model as within-participants factors; gender and lean/obese status were introduced in the model as between-participants factors. Main effects and interactions terms were examined, and effect size was evaluated with the $\omega^{2}$ statistic. For the long-term study, hierarchical linear regression was used to compare the two groups (metreleptin and placebo). Baseline intercepts and slopes between the two groups were compared under the general linear hypothesis. In order to determine the effect of treatment after 32 weeks, we re-centred the time variable at 32 weeks and compared the intercepts of the two groups. Adjustments for oestradiol, 25-hydroxy-vitamin D levels and both were performed in sequential models. Spearman's correlations were performed to examine linear relationships between the pro-angiogenic factors and leptin or other physiological variables. For these correlations we used pooled data from all participants in the long-term study, all time points and both study arms.

\section{Results}

In vitro experiments

Three-dimensional HUVEC angiogenesis model We evaluated 12-17 beads in each treatment group (negative control, metreleptin $50 \mathrm{ng} / \mathrm{ml}$, metreleptin $100 \mathrm{ng} / \mathrm{ml}$ and VEGF $20 \mathrm{ng} / \mathrm{ml}$ ). Leptin did not promote angiogenesis in this three-dimensional angiogenesis model in both doses (50 and $100 \mathrm{ng} / \mathrm{ml}$ ). The sprout count and the cumulative sprout 
length at the metreleptin treated cells were not different from the negative control, whereas treatment with VEGF $(20 \mathrm{ng} / \mathrm{ml})$, as a positive control, significantly increased both the sprout count and the cumulative sprout length, confirming the validity of the assay (Fig. 1).

\section{Clinical studies}

Short-term metreleptin administration study: changes in leptin concentration Serum leptin concentrations peaked $3.43 \pm 1.22 \mathrm{~h}$ after metreleptin injection, to concentrations that were on average 2.5 times higher than baseline when given the physiological dose and 20 times higher than baseline when given the pharmacological dose. Leptin concentrations were significantly higher than baseline at 6 and $12 \mathrm{~h}$ after administration of the physiological metreleptin dose $(0.1 \mathrm{mg} / \mathrm{kg})$ and at 6,12 and $24 \mathrm{~h}$ after administration of the pharmacological metreleptin dose $(0.3 \mathrm{mg} / \mathrm{kg})$. Leptin concentrations remained higher than
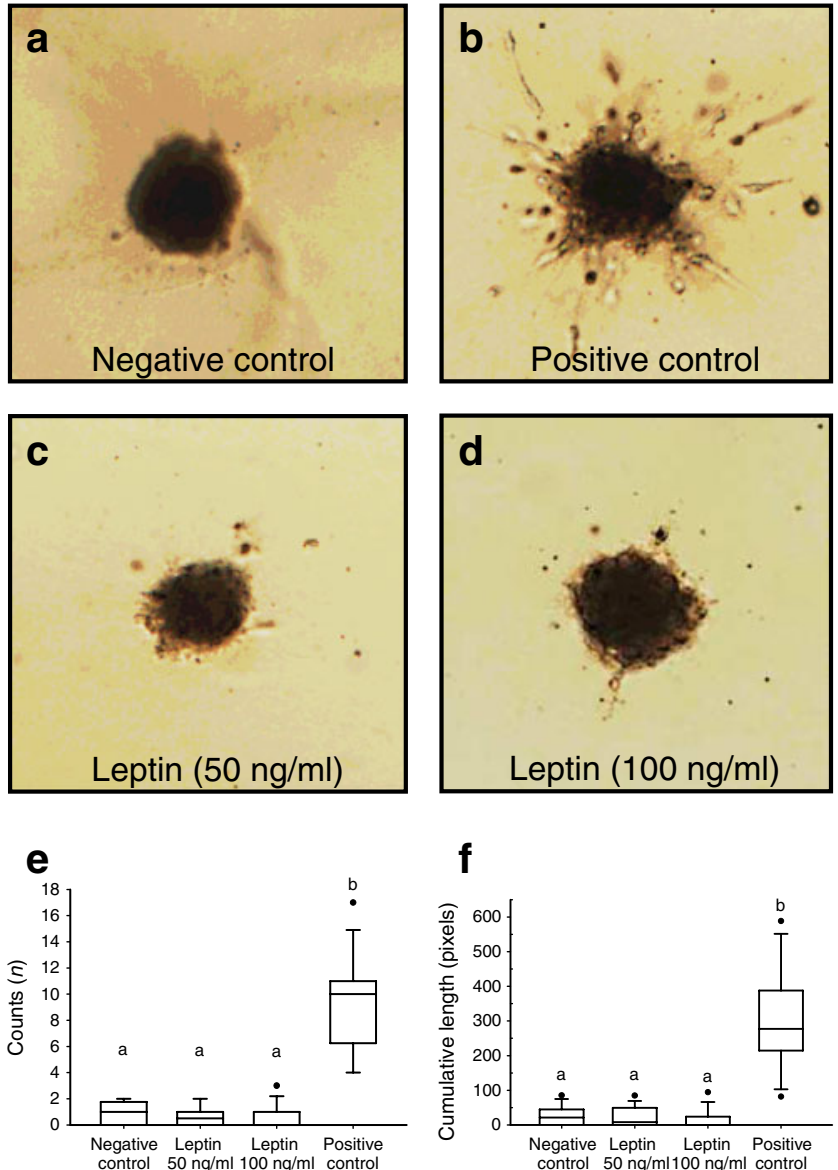

Fig. 1 In vitro three-dimensional HUVEC angiogenesis model. Cells were treated with leptin (50 and $100 \mathrm{ng} / \mathrm{ml}$, c and d) and VEGF (20 ng/ml as positive control, b) for $48 \mathrm{~h}$. a The negative control. e Sprout counts; f sprout cumulative length. ${ }^{\mathrm{a}}$ No statistical significance at the 0.05 level. ${ }^{\mathrm{b}}$ Box plot are statistically different from those marked with ${ }^{\text {a }}$. Statistical outliers are denoted with black circles baseline for at least $12 \mathrm{~h}$ in response to a metreleptin dose of $0.1 \mathrm{mg} / \mathrm{kg}$ and at least $24 \mathrm{~h}$ in response to the $0.3 \mathrm{mg} / \mathrm{kg}$ of metreleptin (Table 1). Baseline leptin concentrations as well as the magnitude and temporal nature of the rise in leptin concentrations after the administration of metreleptin were different among lean men, obese men and lean women. When administered a $0.3 \mathrm{mg} / \mathrm{kg}$ dose of metreleptin, leptin levels peaked $3.60 \pm 0.89 \mathrm{~h}$ after the injection to a concentration of $168.7 \pm 61 \mathrm{ng} / \mathrm{ml}$ in lean men $(n=5)$, whereas in obese men, leptin concentrations peaked $3.4 \pm$ $1.67 \mathrm{~h}$ after the injection to a concentration of $254.3 \pm$ $36.7 \mathrm{ng} / \mathrm{ml}$. In lean women, leptin levels peaked at $2.80 \pm 0.84$ after the injection to a concentration of $440.7 \pm 85 \mathrm{ng} / \mathrm{ml}$. When analysing the entire data with the complete factorial model, gender and lean/obese status had a statistically significant, moderate effect size $\left(\omega^{2}=11.57 \%\right.$ and $18.1 \%$, $p<0.01$ for both) and dose had a statistically significant but mild effect size $\left(\omega^{2}=2.77 \%, p<0.01\right)$.

Proteomic profile analysis The proteomic analysis of pooled sera from the two lean participants revealed that angiogenin and MMP9 signals were stronger $24 \mathrm{~h}$ after metreleptin administration compared with the baseline signal. In the pooled sera from the two obese participants, the angiogenin signal but not the MMP9 signal was higher at the $24 \mathrm{~h}$ time point. The baseline signal of angiogenin was higher in sera from the two obese participants compared with sera from the two lean participants, whereas the MMP9 signal was higher in the sera from the two lean participants. Serpin E1/plasminogen activator inhibitor 1 (PAI-1) and tissue inhibitor of metalloproteinases-1 (TIMP-1) exhibited stronger signals at the $24 \mathrm{~h}$ time point compared with baseline, whereas tissue inhibitor of metalloproteinases-4 (TIMP-4) and u-plasminogen activator (uPA) had weaker signals at the $24 \mathrm{~h}$ time point in the pooled sera from both lean and obese participants (data not shown).

As MMP9 and angiogenin signals differed after metreleptin administration, we decided to further measure the concentrations of these factors with quantifying techniques (described above). We also measured the concentrations of MMP8, ANG-1, PDGF-AA, EGF and VEGF, as these are molecules traditionally associated with angiogenesis (see Discussion). Although we detected some differences in the signals for PAI-1, TIMP-1, TIMP-4 and uPA, we did not proceed to further quantification analysis of these molecules because there is no evidence that associates their circulating levels with angiogenesis in humans.

Angiogenic factors The concentrations of ANG-1, PDGFAA, angiogenin and MMP9 did not change significantly at 6,12 or $24 \mathrm{~h}$ after metreleptin administration for either metreleptin dose (Table 1). We also failed to show any statistically significant change of the aforementioned 
Table 1 Circulating angiogenic factor concentrations: short-term clinical study

\begin{tabular}{|c|c|c|c|c|c|}
\hline Variable & Baseline & $6 \mathrm{~h}$ & $12 \mathrm{~h}$ & $24 \mathrm{~h}$ & $p$ value $^{\mathrm{a}}$ \\
\hline \multicolumn{6}{|l|}{ Lean men $(n=5)$} \\
\hline \multicolumn{6}{|c|}{ Metreleptin dose: $0.1 \mathrm{mg} / \mathrm{ml}$} \\
\hline Leptin (ng/ml) & $2.11 \pm 0.22$ & $52.26 \pm 6.36^{* *}$ & $9.02 \pm 1.13 * *$ & $2.80 \pm .0 .23 * *$ & $<0.01$ \\
\hline Angiopoetin 1(ng/ml) & $27.35 \pm 6.15$ & $19.38 \pm 3.22$ & $23.64 \pm 7.12$ & $4.75 \pm 0.01$ & 0.17 \\
\hline Angiogenin (ng/ml) & $248.96 \pm 24.95$ & $271.73 \pm 28.89$ & $266.84 \pm 45.98$ & $273.61 \pm 32.77$ & 0.66 \\
\hline PDGF-AA (ng/ml) & $2.70 \pm 0.54$ & $2.50 \pm 0.10$ & $2.66 \pm 0.43$ & $0.88 \pm 0.31$ & 0.08 \\
\hline MMP9 (ng/ml) & $225.83 \pm 57.91$ & $154.90 \pm 7.40$ & $209.78 \pm 48.16$ & $41.84 \pm 21.36$ & 0.05 \\
\hline \multicolumn{6}{|c|}{ Metreleptin dose: $0.3 \mathrm{mg} / \mathrm{ml}$} \\
\hline Leptin (ng/ml) & $2.97 \pm 0.30$ & $111.16 \pm 15.08^{* *}$ & $32.36 \pm 5.30^{* *}$ & $4.11 \pm 0.77 * *$ & $<0.01$ \\
\hline ANG-1 (ng/ml) & $29.11 \pm 7.45$ & $18.78 \pm 9.07$ & $16.16 \pm 1.66$ & $28.86 \pm 7.26$ & 0.43 \\
\hline Angiogenin (ng/ml) & $290.89 \pm 5.70$ & $298.72 \pm 25.93$ & $280.76 \pm 10.79$ & $297.72 \pm 18.13$ & 0.76 \\
\hline PDGF-AA (ng/ml) & $2.50 \pm 0.54$ & $1.66 \pm 0.61$ & $2.11 \pm 0.28$ & $2.94 \pm 0.64$ & 0.50 \\
\hline MMP9 (ng/ml) & $348.74 \pm 121.90$ & $188.02 \pm 60.28$ & $148.81 \pm 16.34$ & $194.13 \pm 8.50$ & 0.36 \\
\hline \multicolumn{6}{|l|}{ Obese men $(n=5)$} \\
\hline \multicolumn{6}{|c|}{ Metreleptin dose: $0.1 \mathrm{mg} / \mathrm{ml}$} \\
\hline Leptin (ng/ml) & $15.49 \pm 3.86$ & $83.67 \pm 6.18^{* *}$ & $54.05 \pm 7.05^{* *}$ & $17.95 \pm 2.46^{* *}$ & $<0.01$ \\
\hline ANG-1 (ng/ml) & $13.34 \pm 4.79$ & $2.99 \pm 0.69$ & $32.27 \pm 2.38$ & $17.39 \pm 6.15$ & 0.15 \\
\hline Angiogenin (ng/ml) & $365.38 \pm 42.29$ & $350.39 \pm 16.06$ & $348.77 \pm 41.07$ & $375.82 \pm 75.51$ & 0.83 \\
\hline PDGF-AA (ng/ml) & $2.13 \pm 0.57$ & $0.71 \pm 0.04$ & $4.51 \pm 0.57$ & $2.93 \pm 0.92$ & 0.19 \\
\hline MMP9 (ng/ml) & $268.74 \pm 91.22$ & $67.56 \pm 18.21$ & $427.67 \pm 102.15$ & $324.71 \pm 33.40$ & 0.18 \\
\hline \multicolumn{6}{|c|}{ Metreleptin dose: $0.3 \mathrm{mg} / \mathrm{ml}$} \\
\hline Leptin (ng/ml) & $16.24 \pm 2.24^{* *}$ & $242.94 \pm 17.35^{* *}$ & $114.95 \pm 9.89^{* *}$ & $27.60 \pm 4.57^{* *}$ & $<0.01$ \\
\hline Angiopoetin 1(ng/ml) & $26.54 \pm 9.27$ & $12.54 \pm 6.58$ & $24.81 \pm 2.83$ & $39.72 \pm 9.55$ & 0.23 \\
\hline Angiogenin (ng/ml) & $297.02 \pm 29.82$ & $298.94 \pm 33.22$ & $339.41 \pm 48.31$ & $340.61 \pm 40.42$ & 0.35 \\
\hline PDGF-AA (ng/ml) & $2.87 \pm 0.66$ & $1.70 \pm 0.64$ & $3.00 \pm 0.20$ & $3.89 \pm 0.32$ & 0.28 \\
\hline MMP9 (ng/ml) & $264.88 \pm 51.28$ & $159.85 \pm 38.12$ & $269.16 \pm 71.33$ & $374.47 \pm 104.62$ & 0.29 \\
\hline \multicolumn{6}{|l|}{ Lean women $(n=5)$} \\
\hline \multicolumn{6}{|c|}{ Metreleptin dose: $0.1 \mathrm{mg} / \mathrm{ml}$} \\
\hline Leptin (ng/ml) & $14.36 \pm 4.97$ & $108.71 \pm 13.15^{* *}$ & $25.82 \pm 4.09^{* *}$ & $13.66 \pm 2.85^{* *}$ & $<0.01$ \\
\hline ANG-1 (ng/ml) & $36.79 \pm 6.17$ & $29.29 \pm 6.65$ & $22.16 \pm 6.49$ & $31.41 \pm 5.92$ & 0.34 \\
\hline Angiogenin (ng/ml) & $275.41 \pm 27.52$ & $315.45 \pm 38.88$ & $291.33 \pm 29.35$ & $313.89 \pm 40.50$ & 0.34 \\
\hline PDGF-AA (ng/ml) & $3.47 \pm 0.23$ & $3.04 \pm 0.55$ & $2.42 \pm 0.59$ & $3.31 \pm 0.44$ & 0.33 \\
\hline MMP9 (ng/ml) & $233.98 \pm 38.60$ & $186.38 \pm 24.53$ & $192.52 \pm 39.99$ & $282.74 \pm 76.58$ & 0.45 \\
\hline \multicolumn{6}{|c|}{ Metreleptin dose: $0.3 \mathrm{mg} / \mathrm{ml}$} \\
\hline Leptin (ng/ml) & $12.60 \pm 3.48$ & $318.64 \pm 33.76^{* *}$ & $70.80 \pm 12.70^{* *}$ & $15.55 \pm 3.18^{* *}$ & $<0.01$ \\
\hline ANG-1 (ng/ml) & $39.58 \pm 6.84$ & $40.05 \pm 2.75$ & $25.39 \pm 3.12$ & $24.23 \pm 8.03$ & 0.24 \\
\hline Angiogenin (ng/ml) & $295.85 \pm 36.79$ & $311.73 \pm 41.67$ & $364.94 \pm 54.56$ & $340.46 \pm 48.86$ & 0.16 \\
\hline PDGF-AA (ng/ml) & $3.01 \pm 0.38$ & $3.41 \pm 0.44$ & $2.79 \pm 0.46$ & $2.39 \pm 0.68$ & 0.40 \\
\hline MMP9 (ng/ml) & $290.14 \pm 62.98$ & $362.68 \pm 120.37$ & $316.38 \pm 133.73$ & $459.90 \pm 211.87$ & 0.66 \\
\hline
\end{tabular}

Results are presented as mean $\pm \mathrm{SEM}$

${ }^{\mathrm{a}} p$ value, from overall repeated measurements ANOVA

$* * p<0.01$ compared with baseline

angiogenic factors by individually analysing each group (lean men, obese men, lean women, Table 1). When analysing the entire dataset with the complete factorial model, there was no statistical significant main effect for dose, time and lean/obese status at any molecule; however, gender had a statistically significant, but mild, main effect on all of them (ANG-1: $\omega^{2}=4.79 \%, p=0.02$; PDGF-AA: $\omega^{2}=5.43 \%, p=0.01 ;$ MMP9 $\omega^{2}=4.96 \%, p=0.02$; angiogenin-1: $\omega^{2}=1.44 \%, p=0.01$ ) with their levels being slightly higher in women compared with men (Table 1). 
However the gender $\times$ dose and gender $\times$ time interaction terms were not statistically significant, indicating no differential response of both genders at metreleptin administration.

Long-term metreleptin administration study Serum leptin concentrations increased significantly $(p<0.001)$ in the metreleptin group compared with the placebo group across the selected time points. Both the placebo and the metreleptin groups started from the same leptin levels $(p=$ $0.827)$. The leptin levels in the metreleptin group increased with a rate of $1.3 \mathrm{ng} \mathrm{ml}^{-1}$ week $^{-1}$ ( $p=0.007$ compared with placebo), whereas in the placebo group leptin levels remained stable during the whole study ( $p$ for slope in the placebo group $=0.98$, Table 2). This increase remained statistically significant, even after adjusting for oestradiol and 25-hydroxy-vitamin D levels.

Proteomic profile analysis We performed the angiogenesis proteomic profile array on serum of 10 participants $(n=5$ on the metreleptin and $n=5$ on the placebo arm), comparing baseline levels with levels at 32 weeks. There was no change in the signal between baseline and 32 weeks in both groups for all molecules except ANG-2, endocrine gland derived vascular endothelial growth factor (EG-VEGF), Factor III, EGF, granulocyte/macrophage colony stimulating factor (GM-CSF), IL-1 $\beta$, MMP8, neureglin (NRG)-1 $\beta$ and VEGF, where there was a trend to decrease $(p=0.043)$ over the 32 weeks in the metreleptin group but not in the placebo. Taking into consideration the type I error inflation introduced by examining a large number of molecules, we can safely conclude that the angiogenic profile remained constant over the 32 weeks.

Angiogenic factors ANG-1 $(p=0.75)$, angiogenin $(p=0.56)$, PDGF-AA $(p=0.97)$, MMP8 $(p=0.61)$, MMP9 $(p=0.85)$, EGF $(p=0.86)$ and VEGF $(p=0.82)$ levels did not differ after 32 weeks of treatment between the two groups. Both groups started from similar baseline values for all analytes and the slope of each trajectory was not significantly different in the metreleptin treatment group from the placebo group, which was zero (Table 2). As oestradiol and vitamin D levels could confound the angiogenic potential of the participants, we also evaluated their levels over the time. Oestradiol levels in the metreleptin group were higher by an average of $12.88 \mathrm{pg} / \mathrm{ml}(95 \%$ CI 6.80 $18.97 \mathrm{pg} / \mathrm{ml}, p<0.01$ ), compared with the control group, after 32 weeks of treatment. 25-Hydroxy-vitamin D levels changed in both groups with the same rate of $0.16 \mathrm{ng} \mathrm{ml}^{-1}$ day $^{-1}(p=0.03)$; however, there was no effect of metreleptin treatment on 25-hydoxy-vitamin D levels $(p=0.25)$. For this reason, we adjusted the previously described comparisons for oestradiol and 25-hydroxyvitamin $\mathrm{D}$ levels in sequential statistical models, and the results remained identical for all analytes, except for angiogenin levels, where there was a statistically significant decrease in the metreleptin group compared with the placebo, when adjusting for oestradiol levels $(p<0.01)$ and oestradiol and 25-hydroxy-vitamin D ( $p=0.02$; Table 2$)$.

Pooled data from all participants revealed that none of the concentrations of pro-angiogenic factors were correlated with serum leptin concentrations; however, concentrations of most pro-angiogenic factors correlated with each other, including ANG-1 and PDGF-AA (Spearman's $\rho=0.868, p<0.01$ ), Ang-1 and EGF $(\rho=0.696, p<0.01)$, ANG-1 and VEGF $(\rho=0.576, p<0.01)$, PDGF-AA and EGF $(\rho=0.643, p<0.01)$, PDGF-AA and VEGF $(\rho=0.633, p<0.01)$, MMP8 and VEGF $(\rho=0.310, p<0.05)$, EGF and VEGF $(\rho=0.516, p<$ $0.01)$, MMP9 and EGF $(\rho=0.378, p=0.003)$, MMP9 and VEGF $(\rho=0.631, p<0.001)$, MMP9 and ANG-1 $(\rho=0.563$, $p<0.001)$, MMP9 and PDGF-AA $(\rho=0.620 p<0.001)$, and MMP9 and MMP8 $(\rho=0.447, p<0.001)$.

\section{Discussion}

Since the publication of the first study describing that leptin receptor is produced in human vasculature cells [7] and the implication that leptin could regulate angiogenesis in humans, many studies have been conducted, mainly in vitro and in animal models, examining the potential role of leptin in angiogenesis. In the same study [7], the investigators demonstrate that pharmacological doses of leptin also induce neovascularisation of the rat cornea in vivo, whereas leptin-induced neovascularisation does not occur in corneas of rats lacking functional leptin receptors ( $f a / f a$ Zucker rats), supporting the role of leptin in angiogenesis. In a different study, utilising a retinopathy of prematurity mice model, Suganami et al. demonstrated that leptin overproducing mice demonstrate more pronounced retinal neovascularisation than the wild type, whereas ob/ob mice demonstrate significantly less [19]. In addition, many studies have found that leptin administration enhances wound healing, an angiogenic process, in wild-type [26] and $o b / o b$ mice [27-29] but not $d b / d b$ mice, which lack functional leptin receptors [27]. Whether angiogenesis is the primary mechanism mediating accelerated wound healing by leptin is still uncertain [26-30].

Anti-leptin antibodies have been shown to have angiostatic properties in human hepatocellular carcinoma (HCC) biopsy specimens using the in vivo chorioallantoic membrane (CAM) assay [31]. Similarly, in a rat model of NASH, HCCs developed in normal rats but not in rats lacking leptin receptors. Hepatic neovascularisation and elevated levels of VEGF have also been observed in the normal but not Zucker rats [32]. Consistent with these 
Table 2 Circulating angiogenic factor concentrations: long-term clinical study, metreleptin $(n=10)$, placebo $(n=8)$

\begin{tabular}{|c|c|c|c|c|c|c|c|c|c|}
\hline Variable & & Baseline & 8 weeks & 20 weeks & 32 weeks & $p$ value $^{\mathrm{a}}$ & $p$ value $^{\mathrm{b}}$ & $p$ value $^{\mathrm{c}}$ & $p$ value $^{\mathrm{d}}$ \\
\hline \multirow[t]{2}{*}{ Leptin (ng/ml) } & Metreleptin & $3.67 \pm 0.53$ & $15.98 \pm 3.11$ & $28.48 \pm 9.37$ & $43.40 \pm 15.49$ & $<0.01$ & $<0.01$ & $<0.01$ & $<0.01$ \\
\hline & Placebo & $3.45 \pm 0.74$ & $3.32 \pm 0.93$ & $3.46 \pm 0.79$ & $2.17 \pm 0.38$ & $<0.01$ & & & \\
\hline \multirow[t]{2}{*}{ Oestradiol (pg/ml) } & Metreleptin & $13.87 \pm 1.49$ & $12.82 \pm 1.21$ & $18.99 \pm 2.51$ & $28.53 \pm 6.71$ & & & & \\
\hline & Placebo & $11.81 \pm 1.16$ & $13.3 \pm 2.22$ & $12.65 \pm 1.58$ & $11.14 \pm 0.89$ & 0.25 & & & \\
\hline \multirow[t]{2}{*}{ Vitamin D (ng/ml) } & Metreleptin & $24.53 \pm 2.02$ & $30.76 \pm 0.94$ & $30.62 \pm 2.31$ & $31.00 \pm 3.47$ & & & & \\
\hline & Placebo & $30.11 \pm 1.42$ & $33.57 \pm 3.37$ & $34.20 \pm 3.08$ & $36.84 \pm 3.52$ & & & & \\
\hline \multirow[t]{2}{*}{ ANG-1 (ng/ml) } & Metreleptin & $29.50 \pm 4.24$ & $22.58 \pm 4.04$ & $23.62 \pm 3.65$ & $18.65 \pm 4.15$ & 0.75 & 0.44 & 0.78 & 0.27 \\
\hline & Placebo & $20.52 \pm 4.01$ & $22.53 \pm 4.09$ & $16.33 \pm 4.65$ & $18.51 \pm 4.95$ & & & & \\
\hline \multirow[t]{2}{*}{ Angiogenin (ng/ml) } & Metreleptin & $341.34 \pm 18.21$ & $344.47 \pm 29.77$ & $359.00 \pm 27.16$ & $343.70 \pm 35.40$ & 0.56 & $<0.01$ & 0.87 & 0.02 \\
\hline & Placebo & $374.61 \pm 25.02$ & $366.39 \pm 23.64$ & $370.09 \pm 25.61$ & $365.96 \pm 25.97$ & & & & \\
\hline \multirow[t]{2}{*}{ PDGF-AA (ng/ml) } & Metreleptin & $2.81 \pm 0.36$ & $2.24 \pm 0.45$ & $2.52 \pm 0.32$ & $2.30 \pm 0.42$ & 0.97 & 0.93 & 0.66 & 0.42 \\
\hline & Placebo & $2.33 \pm 0.49$ & $2.07 \pm 0.30$ & $2.18 \pm 0.49$ & $2.16 \pm 0.60$ & & & & \\
\hline \multirow[t]{2}{*}{ MMP8 (ng/ml) } & Metreleptin & $6.13 \pm 1.02$ & $5.94 \pm 1.62$ & $6.69 \pm 1.13$ & $7.51 \pm 2.01$ & 0.61 & 0.71 & 0.58 & 0.37 \\
\hline & Placebo & $6.47 \pm 1.63$ & $4.88 \pm 1.13$ & $5.94 \pm 0.99$ & $9.78 \pm 1.45$ & & & & \\
\hline \multirow[t]{2}{*}{ MMP9 (ng/ml) } & Metreleptin & $157.02 \pm 18.00$ & $143.13 \pm 29.54$ & $123.72 \pm 21.67$ & $156.72 \pm 15.85$ & 0.85 & 0.88 & 0.96 & 0.84 \\
\hline & Placebo & $144.25 \pm 33.43$ & $130.59 \pm 41.61$ & $118.77 \pm 32.33$ & $156.54 \pm 45.34$ & & & & \\
\hline \multirow[t]{2}{*}{ EGF (pg/ml) } & Metreleptin & $63.46 \pm 14.31$ & $40.00 \pm 13.42$ & $44.76 \pm 13.47$ & $70.54 \pm 29.52$ & 0.86 & 0.44 & 0.60 & 0.96 \\
\hline & Placebo & $51.24 \pm 17.63$ & $35.21 \pm 1905$ & $61.52 \pm 26.15$ & $47.70 \pm 18.53$ & & & & \\
\hline \multirow[t]{2}{*}{ VEGF (pg/ml) } & Metreleptin & $133.29 \pm 20.84$ & $125.86 \pm 25.74$ & $133.99 \pm 21.34$ & $116.02 \pm 29.87$ & 0.82 & 0.89 & 0.63 & 0.25 \\
\hline & Placebo & $123.31 \pm 42.52$ & $109.42 \pm 34.47$ & $110.46 \pm 51.09$ & $152.01 \pm 55.69$ & & & & \\
\hline
\end{tabular}

Results are presented as mean \pm SEM

${ }^{a}$ Mixed models: effect of treatment ( $p$ for intercept, with time centred at 32 weeks), unadjusted model

${ }^{\mathrm{b}}$ Mixed models: effect of treatment ( $p$ for intercept, with time centred at 32 weeks), adjusted for oestradiol levels

${ }^{\mathrm{c}}$ Mixed models: effect of treatment ( $p$ for intercept, with time centred at 32 weeks), adjusted for 25-hyrdoxy-vitamin D levels

${ }^{\mathrm{d}}$ Mixed models: effect of treatment ( $p$ for intercept, with time centred at 32 weeks), adjusted for both oestradiol and 25-hydroxy-vitamin D levels

findings, it has been suggested that leptin may accelerate melanoma tumour growth by upregulation of VEGF [33] and may also play a role in the neovascularisation of atherosclerotic lesions [34].

The goal of our study was to evaluate, for the first time in humans, whether leptin has an effect on circulating levels of pro-angiogenic factors. Despite strong evidence for a role of leptin in angiogenesis from in vitro and animal studies, the interventional physiology studies we present herein demonstrate that the circulating concentrations of several pro-angiogenic factors do not change in response to either acute $(24 \mathrm{~h})$ administration of physiological and/or pharmacological doses of metreleptin or in response to chronic (32 weeks) administration of replacement doses of metreleptin to humans. These results suggest that mechanisms regulating angiogenesis in humans may differ from those in animals with regard to the role of leptin. This is apparently similar to other actions of leptin which differ between animals and humans, such as regulation of the autonomic nervous system [35].

Furthermore, we performed a novel in vitro threedimensional angiogenesis assay that enabled us to evaluate the effect of metreleptin treatment on HUVECs in vitro. We demonstrated that leptin does not promote angiogenesis in this model. This is in contrast to the only other available human primary cell in vitro study by Bouloumie et al. [17] demonstrating that leptin promoted angiogenesis. Although we tested the same doses, the HUVEC model that they used differs from ours in terms of the matrix in which the HUVECs were embedded (fibrin vs collagen) and this could be one of the reasons for this discrepancy. In addition to this, in that previous paper [17] there was a discrepancy between the leptin doses that achieved angiogenesis in their in vitro and in vivo models ( $100 \mathrm{ng} / \mathrm{ml}$ vs $0.2-3 \mathrm{~g}$ ). Thus, it is apparently more appropriate to emphasise the need for evaluating the angiogenic effect of leptin in concentrations that are relevant to human physiology and/or could be safely used for therapeutic purposes. The metreleptin doses we have administered in the studies described herein are physiological $[24,25]$ or clearly pharmacological [21-23] as used for therapeutic purposes in humans.

The novelty of our work is that it is the first study that directly examines the effect of metreleptin administration on the regulation of angiogenesis in humans. In addition, a 
major strength of this study is that it involves measurements of serum concentrations of pro-angiogenic factors in both short- and long-term time periods and uses both replacement (long term), physiological and pharmacological (short term) doses of metreleptin. Furthermore, it is an interventional study that allows us to draw conclusions about causality in humans. In the short-term study, the patients were completely standardised in terms of caloric intake and the crossover design of this study allowed us to control for potential confounding.

One limitation of our study is that we could not directly measure angiogenesis at the tissue level in humans and/or collect information about potential changes at the gene expression level. The molecules we chose to investigate, however, are good markers of angiogenesis and are representative of all the steps of angiogenesis. ANG-1 plays an important role in endothelial cell survival, sprouting and tube formation, may reduce inflammation and maintains the integrity of the adult vasculature [36, 37]. Both angiogenin and VEGF induce neovascularisation $[38,39]$, in addition to other important physiological functions, including neuroprotective effects (angiogenin) and endothelial cell maintenance/ survival (VEGF) [38, 39]. PDGF-AA promotes vascular stability and has been found to induce angiogenesis in animal models [40]. The matrix metalloproteinase family, to which MMP8 and MMP9 belong, has been associated with angiogenesis. Specifically, elevated concentrations of MMP8 have been associated with histological markers of vascular invasion and melanoma progression [41]. MMP9 has been more strongly associated with angiogenesis, and is one of the MMPs that contributes to the recruitment of cells and cytokines that stimulate angiogenesis [42]. EGF has been associated with the promotion of endothelial cell proliferation [43] and tumour neovascularisation in certain types of cancer [44-46].

Another limitation of our study is related to the generalisability of the long-term clinical study. We examined the long-term effects of metreleptin administration in hypoleptinaemic women with hypothalamic amenorrhoea and used only physiological replacement doses of metreleptin, which resulted in normal leptin concentrations in these women. It thus does not answer the question of whether metreleptin administration, if administered in pharmacological doses to normoleptinaemic or hyperleptinaemic individuals, would increase the levels of pro-angiogenic factors in the long term. However, we have previously demonstrated that even low physiological doses of leptin replacement can have a dramatic effect on normalising neuroendocrine axes $[24,25]$ in hypoleptinaemic, leptin-sensitive, lean women, who have hypothalamic amenorrhoea. Furthermore, evidence from our short-term study using high doses of metreleptin suggests that metreleptin does not increase circulating levels of pro-angiogenic factors in humans. Long-term interventional studies administering pharmacological doses of metreleptin are needed to study the effect of supraphysiological doses of metreleptin in the long term.

The relatively small sample size is another limitation of this study. However, this sample size has previously been adequate to demonstrate significant changes of the hypothalamic-pituitary gonadal, thyroid and other endocrine axes $[2,3,24,25]$. Thus, the effect of metreleptin on circulating levels of angiogenesis factors is weak, if any. Further interventional studies involving short- and longterm metreleptin administration to hypoleptinaemic, normoleptinaemic and hyperleptinaemic participants are needed to fully elucidate this question.

In summary, this initial translational study failed to show that leptin regulates angiogenesis in humans, in contrast to evidence in the literature suggesting that leptin does so in vitro and in animal models. This is not the only action of leptin that has been reported in vitro and in animal models that could not be replicated in humans. Further studies are required to fully evaluate the mechanisms underlying such discrepancies between preclinical and clinical studies in humans. These, in time, are expected to lead to a better understanding of the physiology of leptin in humans.

Acknowledgements We would like to thank J. Chan for contributing in the early phases of this research, as well the General Clinical Research Center at Beth Israel Deaconess Medical Center and the Core Lab for their assistance. This study was supported by National Institutes of Health - National Center for Research Resources grant M01-RR-01032 (Harvard Clinical and Translational Science Center) and grant number UL1 RR025758. The content is solely the responsibility of the authors and does not necessarily represent the official views of the National Center for Research Resources or the National Institutes of Health. Funding was also received from the National Institute of Diabetes and Digestive and Kidney Diseases grants 58785, 79929 and 81913, and AG032030. Amylin Pharmaceuticals, Inc. supplied metreleptin for this study but had no role in the study design; conduct of the study; collection, management, analysis and interpretation of the data; or the preparation, review or approval of the manuscript.

Duality of interest The authors declare that they have no duality of interest associated with this manuscript.

\section{References}

1. Considine RV, Sinha MK, Heiman ML et al (1996) Serum immunoreactive leptin concentrations in normal-weight and obese humans. N Engl J Med 334:292-295

2. Chan JL, Mantzoros CS (2005) Role of leptin in energydeprivation states: normal human physiology and clinical implications for hypothalamic amenorrhoea and anorexia nervosa. Lancet 366:74-85 
3. Chan JL, Heist K, DePaoli AM, Veldhuis JD, Mantzoros CS (2003) The role of falling leptin levels in the neuroendocrine and metabolic adaptation to short-term starvation in healthy men. J Clin Investig 111:1409-1421

4. Mantzoros CS, Moschos S, Avramopoulos I et al (1997) Leptin concentrations in relation to body mass index and the tumor necrosis factor-alpha system in humans. J Clin Endocrinol Metab 82:3408-3413

5. Mantzoros CS (2000) Role of leptin in reproduction. Ann NY Acad Sci 900:174-183

6. Robertson SA, Leinninger GM, Myers MG (2008) Molecular and neural mediators of leptin action. Physiol Behav 94:637-642

7. Sierra-Honigmann MR, Nath AK, Murakami C et al (1998) Biological action of leptin as an angiogenic factor. Science 281:1683-1686

8. Velnar T, Bailey T, Smrkoli V (2009) The wound healing process: an overview of the cellular and molecular mechanisms. J Int Med Res 37:1528-1542

9. Gordon MS, Mendelson DS, Kato G (2010) Tumor angiogenesis and novel antiangiogenic strategies. Int $J$ Cancer 126:1777-1787

10. Miller JW, Adamis AP, Shima DT et al (1994) Vascular endothelial growth-factor vascular-permeability factor is temporally and patially correlated with ocular angiogenesis in a primate model. Am J Pathol 145:574-584

11. Praidou A, Androudi S, Brazitikos P, Karakiulakis G, Papakonstantinou E, Dimitrakos S (2010) Angiogenic growth factors and their inhibitors in diabetic retinopathy. Curr Diab Rev 6:304-312

12. Szekanecz Z, Besenyei T, Szentpetery A, Koch AE (2010) Angiogenesis and vasculogenesis in rheumatoid arthritis. Curr Opin Rheumatol 22:299-306

13. Moulton KS (2006) Angiogenesis in atherosclerosis: gathering evidence beyond speculation. Curr Opin Lipidol 17:548-555

14. Moulton KS, Heller E, Konerding MA, Flynn E, Palinski W, Folkman J (1999) Angiogenesis inhibitors endostatin or TNP-470 reduce intimal neovascularization and plaque growth in apolipoprotein E-deficient mice. Circulation 99:1726-1732

15. Kitade M, Yoshiji H, Kojima H et al (2008) Neovascularization and oxidative stress in the progression of non-alcoholic steatohepatitis. Mol Med Rep 1:543-548

16. Cao Y (2007) Angiogenesis modulates adipogenesis and obesity. J Clin Investig 117:2362-2368

17. Bouloumie A, Drexler HCA, Lafontan M, Busse R (1998) Leptin, the product of $\mathrm{Ob}$ gene, promotes angiogenesis. Circ Res 83:1059-1066

18. Heida NM, Leifheit-Nestler M, Schroeter MR et al (2010) Leptin enhances the potency of circulating angiogenic cells via src kinase and integrin alpha $\mathrm{v}$ beta 5 implications for angiogenesis in human obesity. Arterioscler Thromb Vasc Biol 30:200-206

19. Suganami E, Takagi H, Ohashi H et al (2004) Leptin stimulates ischemia-induced retinal neovascularization-possible role of vascular endothelial growth factor expressed in retinal endothelial cells. Diabetes 53:2443-2448

20. Park HY, Kwon HM, Lim HJ et al (2001) Potential role of leptin in angiogenesis: leptin induces endothelial cell proliferation and expression of matrix metalloproteinases in vivo and in vitro. Exp Mol Med 33:95-102

21. Chan JL, Wong SL, Mantzoros CS (2008) Pharmacokinetics of subcutaneous recombinant methionyl human leptin administration in healthy subjects in the fed and fasting states: regulation by gender and adiposity. Clin Pharmacokinet 47:753-764

22. Chan JL, Wong SL, Orlova C, Raciti P, Mantzoros CS (2007) Pharmacokinetics of recombinant methionyl human leptin after subcutaneous administration: variation of concentration-dependent parameters according to assay. J Clin Endocrinol Metab 92:23072311
23. Wong SL, DePaoli AM, Lee JH, Mantzoros CS (2004) Leptin hormonal kinetics in the fed state: effects of adiposity, age, and gender on endogenous leptin production and clearance rates. J Clin Endocrinol Metab 89:2672-2677

24. Welt CK, Chan JL, Bullen J et al (2004) Recombinant human leptin in women with hypothalamic amenorrhea. N Engl J Med 351:987-997

25. Chou SH, Chamberland JP, Liu X et al (2011) Leptin is an effective treatment for hypothalamic amenorrhea. Proc Natl Acad Sci USA 108:6585-6590

26. Murad A, Nath AK, Cha ST, Demir E, Flores-Riveros J, SierraHonigmann MR (2003) Leptin is an autocrine/paracrine regulator of wound healing. FASEB J 17:1895-1897

27. Ring BD, Scully S, Davis CR et al (2000) Systemically and topically administered leptin both accelerate wound healing in diabetic ob/ob mice. Endocrinology 141:446-449

28. Frank S, Stallmeyer B, Kampfer H, Kolb N, Pfeilschifter J (2000) Leptin enhances wound re-epithelialization and constitutes a direct function of leptin in skin repair. J Clin Investig 106:501-509

29. Kampfer H, Schmidt R, Geisslinger G, Pfeilschifter J, Frank S (2005) Wound inflammation in diabetic ob/ob mice-functional coupling of prostaglandin biosynthesis to cyclooxygenase-1 activity in diabetes-impaired wound healing. Diabetes 54:15431551

30. Liapakis IE, Anagnostoulis S, Karayannakis A et al (2007) Exogenously-administered leptin increases early incisional wound angiogenesis in an experimental animal model. In Vivo 21:797801

31. Ribatti D, Belloni AS, Nico B, Di Comite M, Crivellato E, Vacca A (2008) Leptin-leptin receptor are involved in angiogenesis in human hepatocellular carcinoma. Peptides 29:1596-1602

32. Kitade M, Yoshiji H, Kojima H et al (2006) Leptin-mediated neovascularization is a prerequisite for progression of nonalcoholic steatohepatitis in rats. Hepatology 44:983-991

33. Brandon EL, Gu JW, Cantwell L, He Z, Wallace G, Hall JE (2009) Obesity promotes melanoma tumor growth role of leptin. Cancer Biol Ther 8:1871-1879

34. Kang SM, Kwon HM, Hong BK et al (2000) Expression of leptin receptor $(\mathrm{Ob}-\mathrm{R})$ in human atherosclerotic lesions: potential role in intimal neovascularization. Yonsei Med J 41:68-75

35. Chan JL, Mietus JE, Raciti PM, Goldberger AL, Mantzoros CS (2007) Short-term fasting-induced autonomic activation and changes in catecholamine levels are not mediated by changes in leptin levels in healthy humans. Clin Endocrinol (Oxf) 66:49-57

36. Kim KE, Cho CH, Kim HZ, Baluk P, McDonald DM, Koh GY (2007) In vivo actions of angiopoietins on quiescent and remodeling blood and lymphatic vessels in mouse airways and skin. Arterioscler Thromb Vasc Biol 27:564-570

37. Tsigkos S, Koutsilieris M, Papapetropoulos A (2003) Angiopoietins in angiogenesis and beyond. Expert Opin Investig Drugs 12:933-941

38. Gao XW, Xu ZP (2008) Mechanisms of action of angiogenin. Acta Biochim Et Biophys Sin 40:619-624

39. Maharaj ASR, D'Amore PA (2007) Roles for VEGF in the adult. Microvasc Res 74:100-113

40. Zhang JH, Cao RH, Zhang Y, Jia TH, Cao YH, Wahlberg E (2009) Differential roles of PDGFR-alpha and PDGFR-beta in angiogenesis and vessel stability. FASEB J 23:153-163

41. Vihinen P, Koskivuo I, Syrjanen K, Tervahartiala T, Sorsa T, Pyrhonen S (2008) Serum matrix metalloproteinase- 8 is associated with ulceration and vascular invasion of malignant melanoma. Melanoma Res 18:268-273

42. van Hinsbergh VWM, Engelse MA, Quax PHA (2006) Pericellular proteases in angiogenesis and vasculogenesis. Arterioscler Thromb Vasc Biol 26:716-728 
43. Bertrand-Duchesne MP, Grenier D, Gagnon G (2010) Epidermal growth factor released from platelet-rich plasma promotes endothelial cell proliferation in vitro. J Periodontal Res 45:87-93

44. Trojan L, Thomas D, Knoll T, Grobholz R, Alken P, Michel MS (2004) Expression of pro-angiogenic growth factors VEGF, EGF and $\mathrm{bFGF}$ and their topographical relation to neovascularisation in prostate cancer. Urol Res 32:97-103
45. Sasaki T, Kitadai Y, Nakamura $T$ et al (2007) Inhibition of epidermal growth factor receptor and vascular endothelial growth factor receptor phosphorylation on tumor-associated endothelial cells leads to treatment of orthotopic human colon cancer in nude mice. Neoplasia 9:1066-1077

46. Li G, Mitra S, Wong AJ (2010) The epidermal growth factor variant III peptide vaccine for treatment of malignant gliomas. Neurosurg Clin N Am 21:87-93 